\title{
Participación ciudadana en la elaboración de las políticas públicas de actividad física y deporte: el caso de Chile Citizen participation in the elaboration of public policies for physical activity and sports: the case of Chile
}

*Franklin Castillo-Retamal, ${ }^{* *}$ Carlos Matus-Castillo, ${ }^{* * *}$ Camilo Vargas-Contreras, ${ }^{* * * *}$ Felipe Canan, ${ }^{* * * * * F e r n a n d o ~}$ Augusto Starepravo, *****Amauri Aparecido Bássoli de Oliveira

*Universidad Católica del Maule (Chile), **Universidad Católica de la Santísima Concepción (Chile), ${ }^{* * *}$ Instituto Profesional DUOC-UC (Chile), ${ }^{* * * *}$ Universidade Estadual do Oeste de Paraná (Brasil), ${ }^{* * * * * \text { Universidade Estadual de Maringá }}$

Resumen. El objetivo del trabajo es presentar los procesos de participación ciudadana que el Estado chileno ha implementado para elaborar la reciente política pública nacional de actividad física (AF) y deporte, considerando el desarrollo histórico de la institucionalidad deportiva del país. Se utilizó la metodología cualitativa descriptiva, utilizando como procedimientos la revisión bibliográfica y la técnica de análisis documental. El trabajo permite indicar que existe un avance en el tratamiento de las políticas públicas en deporte y recreación en Chile, toda vez que en la elaboración de las mismas han participado diferentes carteras ministeriales, organizaciones sociales y personas naturales. Se concluye que, a partir de la incorporación, apropiación y desarrollo del deporte moderno en Chile, se observan esfuerzos desde el aparato público por generar espacios de participación y monitoreo del deporte y sus manifestaciones, teniendo como corolario, la actual Política Nacional de Actividad Física y Deporte, vigente entre los años 2016-2025, inclusive.

Palabras clave: deporte, actividad física, políticas públicas, participación, democracia.

Abstract. The aim of this work is to show citizen participation processes that the Chilean State has implemented to elaborate the current national public policies for physical activity and sports, considering the historical development of the country's sports institutions. The qualitative descriptive methodology was used, performing literature review and documentary analysis technique as the procedures. The work indicates that there is an improvement in the elaboration of public policies in sports and recreation in Chile when different ministerial cabinets, social organizations, and natural persons participated in it. It is concluded that, from the incorporation, appropriation, and development of modern sport in Chile, efforts are observed from the public apparatus towards generating spaces for participation and monitoring of sports and its manifestations, keeping the current National Physical Activity and Sport Policy, in force between 20162025 , as a corollary.

Key words: sport, physical activity, public policies, participation, democracy.

\section{Introducción}

Una forma de comprender el fenómeno deportivo es mediante el análisis de las políticas públicas (PP), las que entregan elementos críticos que alientan no solamente la racionalización, la especialización, la meritocracia y la búsqueda de récords, sino que la incorporación social como parte de sus valores y orientaciones (Segura \& Buarque de Hollanda, 2015). En este sentido, una correcta política deportiva permite eficiencia y eficacia en la utilización de los recursos que dispone cada país, elevando así los estándares de calidad de vida de sus habitantes, a través de la práctica física y/o deportiva (Castillo, Canan \& Starepravo, 2017). Además, cabe señalar que el fomento de la actividad física (AF) se establece en el ámbito de las políticas públicas, toda vez que se trata de un asunto de interés común, atendido a través de decisiones tomadas por la autoridad pública (Valdez, 2015; Flores, 2020), lo que se desprende de un concepto amplio y concebido como un curso de acción dirigido a resolver un problema percibido como público, o bien, acciones que deben satisfacer una demanda social (Canan, Silva, Oliveira \& Starepravo, 2014; Moreno, 2015).

Desde ahí, surge la necesidad de contar con evidencias que permitan reflexionar, criticar y proponer alternativas y sugerencias para este sector de la sociedad, considerando para ello los múltiples componentes y responsables del sistema deportivo, a saber, instituciones públicas, gobiernos

Fecha recepción: 21-11-19. Fecha de aceptación: 03-03-20 Franklin Castillo-Retamal

fcastillo@ucm.cl locales, organizaciones privadas con fines de lucro y las del tercer sector, que se desarrollan en las diversas dimensiones del deporte: recreativo, formativo, competitivo y de alto rendimiento, que en Chile están consideradas en la Ley del Deporte (Chile, 2001). De acuerdo con esto, las prioridades que establecen los gobiernos en el ámbito deportivo comprenderían las políticas de Estado para este sector, las que deben desarrollarse de forma articulada colectivamente por el mismo, sumando a este desarrollo a los otros sectores públicos y privados de la sociedad (De Quadros Martins, 2011; Canales-Lacryz \& Rey-Cao, 2018).

En Chile, el estudio de las políticas públicas del deporte y AF, es y ha sido un campo de análisis escasamente abordado, según Bravo \& Silva (2013), recién desde la década de 1990 los gobiernos chilenos han mostrado interés más explícito por el deporte. Actualmente, existen pocas evidencias documentadas en esta área, es el caso de los trabajos de Bravo \& Silva (2013); Sandoval \& García (2014); Moreno \& Poblete (2015); Soto-Lagos (2016; 2018); Castillo, Canan \& Starepravo (2017) y Vargas, Matus \& Duclos (2020), no obstante, se observa una corriente incipiente de trabajos de finalización de grado académico, ubicados en niveles de estudio de licenciatura y postgrados (Valdebenito, 2013; Escobar \& Rivas, 2013; Jofré, 2014; Vargas, 2015; Badilla \& Kemnis, 2016; Barría, 2017; Taibo \& Palacio, 2017), lo que indicaría una clara inquietud por emprender en un ámbito poco estudiado, lo que sería coherente al considerar los importantes cambios en las estructuras institucionales y mecanismos de planeación que se han producido en esta materia durante los últimos años.

No obstante, surge la inquietud de conocer la motiva- 
ción del Estado para promover la creación de una nueva PP del sector deportivo y la $\mathrm{AF}$, considerando que históricamente estas áreas de desarrollo han estado postergadas a un segundo y tercer lugar de prioridad. En este sentido, son diversos los informes y diagnósticos elaborados durante los últimos años, los que entregan un panorama negativo a nivel nacional y regional (Encuesta Nacional de Hábitos de Actividad Física y Deportes en Población de 18 años y más (2018); Encuesta Nacional de Salud (2017); Encuesta de Actividades de Niños, Niñas y Adolescentes (2012); Estudios Nacionales de Educación Física (2015), entre otros), evidenciando por ejemplo, los altos niveles de sedentarismo e incumplimiento de las recomendaciones de AF promovidas por la Organización Mundial de la Salud; escasa adherencia a la práctica deportiva en organizaciones formales (clubes, ligas, asociaciones, etc.) y menor participación de la mujer en relación al hombre en la práctica deportiva, entre otros resultados. Lo anterior, se puede asociar con la potencial relación y las propiedades que se le otorgan generalmente a la práctica de AF respecto a su rol como agente preventivo y de intervención ante el sobrepeso, la obesidad, el sedentarismo y en el mejoramiento de la seguridad pública, reducción del tabaquismo, alcoholismo y otras drogas. Cabe mencionar que la PP anterior (Chile, 2002), no tuvo mayores cambios y/ o actualizaciones en función del nuevo escenario institucional y social del país y, a la vez, fue elaborada con escasas posibilidades y espacios de participación para la sociedad civil y organizaciones públicas.

Basados en estas consideraciones, se busca en las próximas líneas atender al objetivo de presentar los mecanismos de participación ciudadana que el Estado chileno ha implementado para elaborar la reciente política pública nacional de actividad física (AF) y deporte, considerando el desarrollo histórico de la institucionalidad deportiva del país.

Para el desarrollo de este trabajo, nos hemos posicionado desde un enfoque cualitativo (Flick, 2015), tomando dos vertientes para su desarrollo, una exploratoria y otra descriptiva (Gil, 1991), considerando en primer lugar que las PP del deporte son un espacio escasamente estudiado en el contexto nacional y latinoamericano; y en segundo lugar, una línea descriptiva, referente al análisis y características propias del objeto de estudio.

Como procedimiento, se utilizó la recopilación y selección bibliográfica (Gil, 1991) para luego emplear la técnica de análisis documental (Quivy \& Campenhoudt, 2006), toda vez que permite analizar metódicamente informaciones que presentan un alto grado de profundidad y complejidad. La selección del material bibliográfico se basó en las referencias existentes de las relaciones entre el deporte, las organizaciones, las PP del sector y el Estado. Respecto a los documentos empleados, se recurrió a instrumentos legales y normativos, herramientas de planeación y artículos publicados.

\section{Las políticas públicas y la incorporación de la participa- ción ciudadana}

El análisis de las PP permite aportar a la discusión sobre temas como democracia, participación, organización y gestión, cuestión indispensable para quienes procuran reflexionar responsablemente sobre política (Frey, 2000; Roth, 2014).
En esta línea Rodríguez (2017), sostiene que en todas las definiciones de políticas públicas existe la participación gubernamental, las demandas sociales, la orientación a resolver nudos problemáticos y la revisión de las prioridades para la elaboración y ejecución de las mismas. En definitiva, es posible indicar que las políticas públicas son elaboradas por los gobiernos con la finalidad de resolver demandas sociales específicas, teniendo como camino los elementos legales para su logro. Sin embargo, establecer indicadores como la eficacia y eficiencia de los mismos, debe ser parte de la observación permanente en todas las fases, vale decir, desde su concepción hasta la ejecución.

En este marco, la participación ciudadana en la gestión de las PP no es un enfoque nuevo en el campo de la ciencia política, pero ha tomado mayor relevancia en las últimas décadas a raíz de la crisis de representatividad de los sistemas políticos formales. Las explicaciones frente al fenómeno son diversas, Beck (2004) por ejemplo, atribuye responsabilidades a los procesos de globalización en la pérdida de capacidades de asociación colectiva de la población, a la vez, ha puesto en crisis el campo de acción del aparato gubernamental estatal, desarticulando la participación social y política, aumentando la deslegitimación de las instituciones públicas formales, por ende, el descontento ciudadano frente a la mejoría en las condiciones de vida.

Así, la participación ciudadana emerge como un principio y enfoque obligatorio en la gestión pública, instalándose con mayor fuerza en las discusiones en torno a la formulación de PP territoriales, vale decir, pasan a ser actor clave en la generación de proyectos de desarrollo e inversión pública para la misma comunidad (Sabatini, Arenas \& Núñez, 2011).

Mujica (2005), sostiene que la participación ciudadana o comunitaria, no solo beneficia las metas, sino también es una vía de acción, que por sí sola desarrolla el capital social de la comunidad. Los aspectos centrales de ese capital conformado por los grados de confianza, la capacidad de asociatividad, el compromiso cívico y los valores éticos positivos crecen con la participación. En otras palabras, es una forma de entender el ejercicio del poder, donde los ciudadanos forman parte activa de la gestión pública, transformándose así en un derecho ciudadano fundamental y prerrequisito para el ejercicio de otros derechos (Corporación Participa, 2008).

La participación ciudadana ofrece la oportunidad de desarrollar y fortalecer la gobernabilidad al generar canales de comunicación mayormente horizontales entre la autoridad, los gobiernos locales y las personas, generando la capacidad y recursos para responder a las necesidades de los ciudadanos (PNUD, 2004; Díaz, 2017; Marcen, Murillo, Arbones \& Pelegrín, 2019).

En el contexto normativo nacional, es la Ley 20.500 (Chile, 2011), la que formaliza este enfoque y entiende la participación ciudadana como un proceso de cooperación, mediante la cual el Estado y la ciudadanía identifican y deliberan conjuntamente acerca de problemas públicos y sus soluciones, con metodologías y herramientas que fomentan la creación de espacios de reflexión y diálogo colectivos, encaminados a la incorporación activa de la ciudadanía en el diseño y elaboración de las decisiones públicas. De esta forma, se manifiesta la intención del Estado de permitir la inclusión paulatina de la sociedad civil en la gestión de las PP del país, 
estableciendo para ello mecanismos específicos bajo la tutela de cada servicio público.

Las políticas públicas del deporte: ¿¿una demanda de las sociedades contemporáneas?

Las PP en AF y deporte, se han caracterizado a lo largo del tiempo por no estar en la línea de prioridades de los Estados y gobiernos, donde sí es posible observar avances en otras áreas como salud, educación y seguridad social, lo que indudablemente se encuentra determinado e influido por el contexto del desarrollo social y económico (Segura \& Buarque de Hollanda, 2015). No obstante, considerando que la AF y el deporte no son prioridades nacionales, es relevante identificar qué nos aporta el diseño y concepción de correctas PP en el área. Para este caso, Tejero-González (2016) postula cuatro razones que justificarían al deporte como una acción política: 1) incrementa el bienestar físico, mental y social de los ciudadanos, mejorando la calidad de vida e incidiendo en el estado de bienestar; 2) facilita la unidad y la cohesión de la sociedad; 3) promueve mayoritariamente los valores y principios de las sociedades democráticas; 4) e es una oportunidad de impulso y reactivación económica.

El deporte en el contexto actual, comprendido desde todos sus niveles de desarrollo, se ha establecido en la discusión y análisis de importantes organismos a nivel internacional, los cuales, en la Declaración de Berlín (UNESCO, 2013) reconocen que para su desarrollo y sustentabilidad es necesario, por ejemplo, contar con políticas públicas en las cuales participen todos los actores sociales. En este mismo sentido, la Carta Internacional de la Educación Física, la Actividad Física y el Deporte (UNESCO, 2015), manifiesta que todas las partes interesadas deben participar en la creación de una visión estratégica que determine las opciones y prioridades en materia de políticas, destacando así el sentido participativo en el diseño de las políticas públicas.

Otros componentes relevantes para la elaboración de cualquier PP son los datos (la evidencia), los que se materializan a partir de las informaciones que disponemos para identificar los problemas y proponer soluciones. La Carta Internacional de la Educación Física, la Actividad Física y el Deporte (UNESCO, 2015), en su artículo $6^{\circ}$, manifiesta que la investigación, los datos empíricos y la evaluación son elementos fundamentales para el desarrollo del deporte y la Educación Física (EF). Junto a ello, la Declaración de Berlín (UNESCO, 2013), expone la necesidad de que las informaciones se transmitan mediante procesos de intercambio a nivel nacional, regional e internacional, lo cual puede acrecentar la eficacia y la sostenibilidad de las políticas respectivas. En tal sentido, es necesario que toda PP diseñada y aplicada debe tener mecanismos de evaluación sistemáticos con el objetivo de saber en qué medida responden a las necesidades de los beneficiarios previstos, esto, entendiendo que la aplicación de políticas públicas por sí misma no garantiza necesariamente resultados positivos.

\section{Deporte y política pública en Chile: antecedentes histó- ricos}

De acuerdo a la propuesta cronológica de Escobar \&
Rivas (2013), en el año 1889 se dicta un Decreto de Ley que hace obligatoria la enseñanza de la EF en los recintos educacionales, o sea, es posible decir que los orígenes legales de la organización institucional pública relacionada al deporte en Chile nacen a partir de la EF (Castillo, Canan \& Starepravo, 2017). En el año 1906 se crea por Ley el Instituto de Educación Física y Manual de la Universidad de Chile y en 1918, según Decreto $\mathrm{N}^{\circ} 4558$, se aprueba el reglamento de este Instituto, el que también cambia de nombre a Instituto Superior de Educación Física y Técnica, el que tenía por objeto formar docentes en las áreas de Educación Física, Deportes y Técnica. En 1923, se promulga el decreto $N^{\circ} 1574$ sobre la Comisión Nacional de Educación Física, teniendo su reglamento el mismo año, transformándose así en el primer organismo público de fomento deportivo creado para organizar y planificar la política estatal nacional en materia de Educación Física. En 1942, se dicta la Ley N 7.200, que crea la Dirección de Informaciones y Cultura, teniendo un Departamento de Deportes, creado al año siguiente por Decreto $\mathrm{N}^{\circ} 35 / 6331 \mathrm{de}$ 1942 (Chile, 1942).

En los siguientes veinte años, el Estado participa más activamente en el campo de la EF y el Deporte de acuerdo al gobierno de turno, por ejemplo, la refundación del Departamento de Deportes en el año 1948 y de la Comisión de EF y Deportes en 1954. En el gobierno comprendido entre los años 1964-1970, surge la relación entre el Ministerio de Educación y la Dirección General de Deportes, carteras que trabajarían en conjunto (Muñoz, 2001), vislumbrándose con ello, algunas características del actual estado de la EF en Chile (Cornejo, Matus \& Vargas, 2011; Castillo, Almonacid, Castillo \& Oliveira, 2020).

En 1970, se promulga la Ley $\mathrm{N}^{\circ}$ 17.276 (Chile, 1970), que aprueba las normas para el fomento del deporte, donde, según Muñoz (2001), el poder ejecutivo contaba con una agenda deportiva que tenía entre sus tareas modificar estructuralmente la normativa pública deportiva, entre ellas, rediseñar la Dirección de Deportes del Estado y crear un Consejo Nacional del Deporte, organismo orientado a dirigir la EF escolar, extraescolar y militar, el deporte y el esparcimiento, como una forma de aportar a la actividad deportiva. Esta Ley se promulgó el 05 de enero de 1970 y, en su primer párrafo, se refería a la Dirección General de Deportes y Recreación (DIGEDER) y a la Comisión Nacional Asesora, las que dependían del Ministerio de Defensa Nacional a través de la Subsecretaría de Guerra (Matus, Vilanova, Puig \& Vidal, 2018).

En el gobierno comprendido entre los años 1970 y 1973 , se diseñaron una serie de programas que fomentaban disciplinas como el atletismo, gimnasia, vóleibol, básquetbol y natación. También tuvo cabida el deporte laboral y, en ese periodo, se llevaron a cabo los Primeros Juegos Laborales organizados por la Central Unitaria de Trabajadores (CUT) y la DIGEDER. La preocupación por el trabajador no sólo estuvo en este evento, sino también se les dio la posibilidad de capacitación a los interesados para transformarse en instructores deportivos calificados.

En 1971, según Decreto № 369 del Ministerio de Defensa Nacional (Chile, 1971), se aprueba el reglamento sobre funcionamiento, organización y atribuciones de los Consejos Locales y Provinciales de deporte, que caracteriza a los Con- 
sejos Locales de Deporte (COLODEP), como organismos autónomos cooperadores del Estado en la función de fomentar el deporte, coordinar actividades deportivas locales y la administración de los recintos deportivos. Cabe mencionar que, durante este periodo, se oficia un artículo denominado «Filosofía de la Nueva Política del Gobierno Popular», el que postulaba que era un derecho de todos los chilenos tener acceso a actividades educativo-físicas y deportivorecreativas, lo que se constituyó en una de las preocupaciones del Estado (Matus et al., 2018).

Durante el gobierno militar(1973-1989), el Ministerio de Defensa Nacional (1975), fija las orientaciones fundamentales por las que se debía regir el deporte y la recreación en Chile. Se estructuró el deporte a través del Plan Nacional de Deportes y Recreación (PLANDYR) y el Sistema Nacional de Deportes y Recreación (SINDYR), los que tenían una proyección de diez años y una meta de $33 \%$ en cuanto al porcentaje de cobertura poblacional (Muñoz, 2001).

En la década de 1990 (1996), el gobierno presentó al Congreso Nacional un proyecto que creaba una Ley del Deporte, el cual fue aprobado por ambas cámaras (Diputados y Senadores) luego de pequeñas modificaciones, pero conservando el texto primitivo confeccionado por el ejecutivo (Escobar \& Rivas, 2013), sin embargo, según Bravo \& Silva (2013), este proyecto se transformó en Ley en el año 2001 bajo una nueva gestión presidencial.

Durante el gobierno comprendido entre los años 2000 y 2006, se promulga el año 2001, la Ley del Deporte $N^{\circ} 19.712$, suprimiendo así la Dirección General de Deportes y Recreación (DIGEDER), que se sustituye por el Instituto Nacional de Deportes (IND), conocido también como Chiledeportes. También ese mismo año, se promulga el Decreto $\mathrm{N}^{\circ} 59$, que versa sobre el Reglamento de Organizaciones Deportivas que está actualmente vigente (Chile, 2001). El programa de becas para deportistas de alto rendimiento del IND, fue el último reglamento promulgado por este gobierno en materia deportiva a través del Decreto $\mathrm{N}^{\circ} 42$ de enero de 2006.

Así mismo, en el año 2002, se definió la Política Nacional de Actividad Física y Deporte - PNAFYD (2002-2014), documento que estableció el marco en que debían orientarse los esfuerzos como país en términos de promoción, masificación y mejoramiento del quehacer deportivo (Chile, 2002). A partir de esto, se elaboró un plan estratégico que presentaba los siguientes objetivos: 1) ampliar la cobertura, fortalecer los programas y la calidad de las actividades físicas y deportivas en la comunidad nacional; 2) incorporar y fortalecer a los grupos organizados en el desarrollo y ejecución de programas permanentes de actividad física y deportiva; 3) posicionar los valores y beneficios de la práctica de la actividad física y deporte en la población y; 4) desarrollar y mejorar el nivel y posición del deporte de alto rendimiento nacional en competiciones internacionales. En este periodo, es posible observar una inyección importante de recursos e inversión pública en relación a la masificación del deporte, donde la categoría de infraestructura toma la delantera (Campeonato Mundial Femenino de Fútbol sub-20 en 2008, Juegos Deportivos Sudamericanos y Parasudamericanos en 2014 y el más reciente, Campeonato Mundial Masculino de Fútbol sub-17 el año 2015).

El gobierno que ejerce entre los años 2006 y 2010 promul- ga el Decreto $\mathrm{N}^{\circ} 75$ (Chile, 2006), que trata sobre el reglamento para organizaciones deportivas profesionales. La única iniciativa que se dictó con rango de Ley fue la $\mathrm{N}^{\circ} 20.178$ (2007), que regula la relación laboral de los deportistas profesionales y trabajadores que desempeñan actividades conexas. Durante este periodo, se observó por primera vez en la historia del país, una intención concreta por parte del ejecutivo de tratar la actividad deportiva de forma seria, al presentar la iniciativa de crear un Ministerio que se dedicase a estos menesteres.

Durante el periodo presidencial comprendido entre 2010 y 2014, la obra legislativa más importante es la creación del Ministerio del Deporte (MINDEP) (que ya se había presentado como proyecto en 2007), cartera que se encuentra vigente desde agosto de 2013 a través de la Ley 20.686 (Chile, 2013).

La agenda del gobierno 2014-2018 a través del MINDEP, desarrolló desde 2015, estrategias de participación comunitaria en virtud de concordar en la elaboración de nuevas políticas públicas en materia de deportes, AF y recreación, donde los actores y beneficiarios se hacían parte y pieza fundamental en el levantamiento de propuestas para el desarrollo de actividades de acuerdo a los contextos y necesidades propias de cada región.

En esa línea, una de las acciones concretas fue la elaboración de las Recomendaciones para la Práctica de Actividad Física según curso de vida (Chile, 2017), a partir de un convenio de colaboración entre los Ministerios de Deporte, Salud y Educación, donde un grupo de académicos expertos entregan orientaciones generales respecto a la actividad física para la comunidad de acuerdo a la etapa de vida. La metodología utilizada en este caso, fue el establecimiento de mesas técnicas de trabajo según etapa de vida, emergiendo de esa discusión, orientaciones que se fundamentaron en las características de cada grupo etario. Una condición distintiva e importante, es el carácter intersectorial del trabajo, que incluyó representantes de cada uno de los ministerios individualizados anteriormente.

Por otra parte, la PNAFYD 2016-2025 (Chile, 2016), que fue coordinada a partir de 2015 por el MINDEP, contó la participación y colaboración de los Ministerios de Educación, Salud, Vivienda y Urbanismo además del Ministerio de Desarrollo Social, teniendo como objetivo principal, promover hábitos de vida saludable y aumentar la práctica física en la población, de tal forma de mejorar la calidad de vida de la comunidad.

A la luz de los antecedentes, se observa un avance en el tratamiento de las políticas públicas en deporte y recreación en Chile, entregando al país algunos rasgos en relación a la cultura deportiva nacional, toda vez que en la elaboración de las mismas han participado desde carteras ministeriales hasta las organizaciones sociales a lo largo del país. Al mismo tiempo, es posible establecer que, a partir de las acciones desplegadas en torno a la política deportiva, se observan diferentes énfasis donde el denominador común es el deporte en sus diferentes manifestaciones y niveles. Por otro lado, también se orienta la política hacia el desarrollo social, cuestión altamente valorada por los segmentos sociales que ven en su despliegue la preocupación del Estado por el bienestar de la población. 


\section{Incorporación de la participación ciudadana en las polí- ticas públicas deportivas}

La participación ciudadana se constituye en una herramienta ambivalente al momento de diseñar políticas para el sector del deporte y la $\mathrm{AF}$, entendiendo que la inclusión social en la toma de decisiones marca un desafío en la gestión de convocatorias. Muñoz (2001), señala que los gobiernos adquieren conciencia de la necesidad pública que origina la práctica deportiva cuando constatan que, tanto aficionados como practicantes, se organizan en clubes y asociaciones, que coordinan sus actividades con federaciones nacionales e internacionales y que tienen la capacidad de convocar y celebrar eventos deportivos con otras localidades. Así se empieza a producir la necesidad de jerarquizar la acción del Estado chileno en la materia, no solo asumiendo compromisos en su promoción y financiamiento, sino también promoviendo y tutelando la creación de nuevas instituciones que representen los intereses deportivos de la población(Vargas, 2015).

Durante los años 2015 y 2016, el MINDEP desarrolló un proceso de formulación de la PNAFYD2016-2025 (Chile, 2016), que actualiza el instrumento impulsado en el año 2001 por el IND como resultado del trabajo participativo entre distintos actores y sectores que toman parte en forma directa e indirecta en el desarrollo deportivo y de la AF. El principal mecanismo para llevar a cabo este proceso fueron los diálogos participativos, definidos como espacios de vinculación entre la ciudadanía y las autoridades del Estado en torno a temas de interés público.

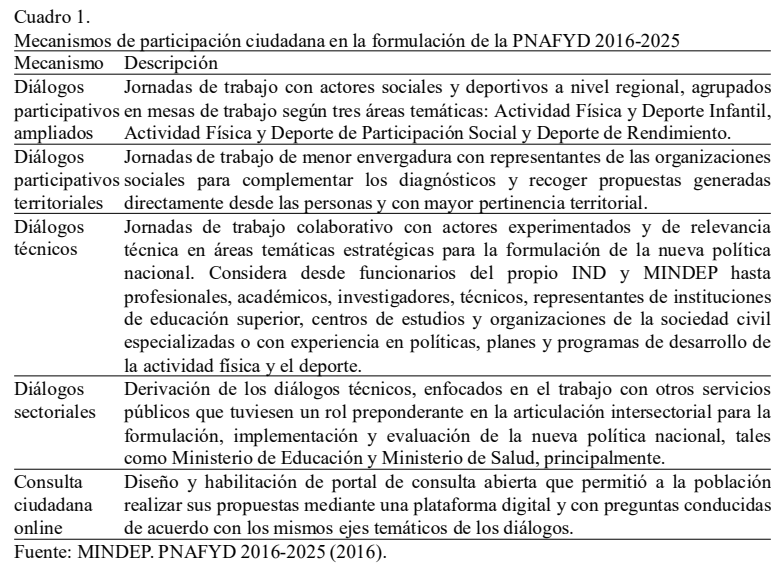

De acuerdo con datos del MINDEP (Chile, 2016), el proceso de levantamiento de información alcanzó una participación efectiva de 12.918 personas en los 231 diálogos ciudadanos, ampliados y territoriales, realizados a nivel nacional y en las 15 regiones del país, de los cuales 7.610 (59\%) fueron hombres y 5.308 (41\%) mujeres. A esta cifra, se le suman 1.616 personas participantes de diálogos técnicos y sectoriales que permitieron el levantamiento de propuestas de trabajo con mayor especificidad.

\begin{tabular}{lcc} 
Cuadro 2. \\
Participación ciudadana en la formulación de la pnafyd 2016-2025 \\
\cline { 2 - 3 } Criterio & Política de actividad física y deporte \\
\hline Actividades ciudadanas & 2002 & 2016 \\
Cobertura regional & 11 & 231 \\
Cobertura comunal & 8 & 15 \\
Participación & 8 & 208 \\
Tiempo formulación & 881 personas & 14.534 personas \\
\hline FUENTE: MINDEP. PNAFYD $2016-2025(2016)$ & 36 meses
\end{tabular}

Si bien la cobertura del proceso técnico y la representatividad ciudadana en el levantamiento de información son aspectos diferenciadores respecto a la formulación de la Política Nacional del año 2002 a cargo del IND, uno de los aspectos más relevantes del diseño metodológico de formulación de esta nueva política es la articulación territorial de sus productos estratégicos, mediante la definición de etapas de trabajo entre los años 2015 y 2017, que buscan generar coherencia entre las definiciones de la política nacional, con lo que puedan determinar las regiones y las comunas a través de sus propios instrumentos de gestión, como las políticas regionales, a cargo de los Gobiernos Regionales y los planes comunales, a través de la coordinación de los municipios y las organizaciones territoriales, respectivamente.

\begin{tabular}{|c|c|c|}
\hline \multirow[b]{2}{*}{ Criterio } & \multicolumn{2}{|c|}{ Política de Actividad Física y Deporte } \\
\hline & 2002 & 2016 \\
\hline \multirow[t]{9}{*}{ Productos estratégicos } & Política Nacional & Etapa 1 (2015-2016) \\
\hline & 13 Politicas Regionales & Política Nacional \\
\hline & & Plan Nacional \\
\hline & & Etapa 2 (2016-2017) \\
\hline & & 15 Políticas Regionales \\
\hline & & 15 Planes Regionales \\
\hline & & Etapa 3 (2016 en adelante) \\
\hline & & Asesoría en Planes Comunales \\
\hline & & Diseño de Programas sociales \\
\hline
\end{tabular}

La implementación de este diseño metodológico contempló el trabajo directo con las organizaciones de base desde el nivel comunal, ofreciendo a las comunidades locales una estructura de planificación para los planes comunales de desarrollo del deporte y la actividad física que pudiesen articularse temáticamente con las directrices de la política nacional. Para llevarlo a cabo, el MINDEP implementó el Programa «Planes Comunales de Actividad Física y Deporte», consistente en asesoría técnica directa a un conjunto de comunas anualmente, de tal manera que permitiera orientar su trabajo para recoger las demandas ciudadanas y traducirlas en líneas de acción de impacto local.

\section{Reflexiones en torno a la participación ciudadana}

Los diálogos desarrollados con la ciudadanía y coordinados por el MINDEP, permitieron identificar discursos comunes respecto a los problemas que visualiza la población en el desarrollo del deporte y la AF a nivel nacional, regional y local. En este aspecto, Vargas, Matus \& Duclos (2020), proponen un esquema de sistematización de las principales

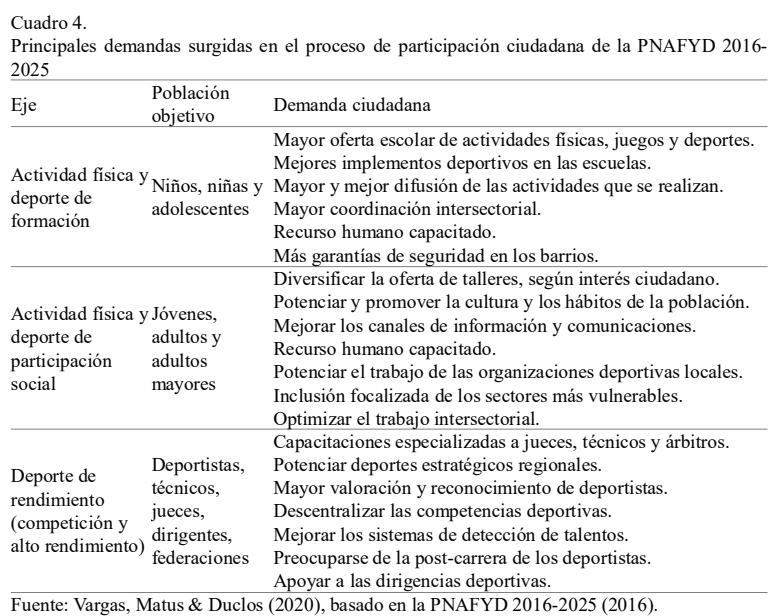


demandas ciudadanas que expone la PNAFYD 2016-2025, con enfoque comunitario y en relación con el fortalecimiento de los hábitos saludables y la calidad de vida de las personas.

El siguiente cuadro toma de referencia el esquema conceptual de los autores e incorpora las demandas ciudadanas en materia de deporte competitivo y de alto rendimiento, a fin de visualizar las problemáticas de la población en los tres ejes estratégicos que establece la política nacional:Los autores señalan que, si bien esta política fue formalizada mediante el Decreto presidencial №31 en abril de 2017 (Chile, 2017), desde el Estado son diversos los instrumentos de fomento de la AF y el deporte que han permitido expresar las PP en planes, programas y proyectos que a la fecha ya cuentan con presupuestos determinados y que pretenden impactar a una determinada población como beneficiarios directos.

Dicho esto, la nueva PNAFYD 2016-2025 tiene el desafío de implementar las nuevas líneas de acción que declara en coherencia con las demandas ciudadanas, pero también lograr una coordinación intersectorial efectiva que permita revisar y actualizar los actuales programas públicos en desarrollo, de tal forma de darle coherencia, pertinencia y sostenibilidad a este instrumento de gestión en el período establecido.

\section{Consideraciones finales}

A razón de la incorporación, apropiación y desarrollo del deporte moderno en la sociedad chilena desde mediados del siglo XIX hasta nuestros días, se observan diversos esfuerzos desde el aparato público por generar espacios y mecanismos de participación y monitoreo del deporte, la AF y la Educación Física. Es a partir de esta disciplina de la educación, desde donde se pesquisan las primeras acciones que se asocian a la intervención del Estado en la gestión pública del deporte, lo que se acompaña por el surgimiento de diversas organizaciones estatales.

La historia política de Chile ha influenciado el desarrollo, no solo de la sociedad, su cultura y la economía, sino que también ha afectado a las instituciones públicas y privadas, en este sentido el deporte y sus organizaciones no han quedado al margen (Matus et al., 2018). Esto se aprecia al observar el recorrido histórico de las acciones políticas en torno al deporte, siendo más fértiles y significativas en las etapas democráticas, no así en los regímenes autoritarios y dictatoriales.

Desde el retorno a la democracia en 1990, el Estado de Chile ha potenciado el progreso de la institucionalidad pública del deporte, jerarquizando su rol y otorgando mayores recursos a las organizaciones públicas del área, estableciendo como eje relevante el trabajo intersectorial de los ministerios interesados. Este desarrollo se ha gestado a la par de acciones en el ámbito normativo, legislativo, político y de planeación para el sector deportivo.

No obstante, de acuerdo al análisis retrospectivo de este trabajo, se observa en Chile un dilatado proceso de instalación y desarrollo de sus PP del deporte, marcadas por la irregularidad y el bajo impacto durante gran parte del siglo $\mathrm{XX}$, caracterizadas más bien por políticas de orden institucional, es decir, creación de nuevas institucionalidades como Chiledeportes (2001), el MINDEP (2013) y normativas, que han comenzado a fijar las bases de regulación del fomento a la AF y deporte, tales como reglamentos de organizaciones deportivas, becas para deportistas, fondos concursables y otros.

A pesar del tránsito intermitente del desarrollo del deporte y la AF desde la intervención pública, se reconocen avances significativos desde el año 2002, con la formalización de la primera política nacional de actividad física y deportes y, el año 2016, con una nueva y actualizada política que establece las principales acciones estratégicas que el MINDEP debe coordinar con actores públicos y privados.

Esta investigación reconoce al menos dos importantes avances para el sector que, en sintonía con la jerarquización del rol del Estado a través del fortalecimiento institucional y la modernización de las estructuras que coordinan el aparato público, permiten ir posicionando las políticas públicas del sector en una dimensión de incipiente formalidad que puede significar una mayor sostenibilidad en el tiempo.

Primero, la incorporación de enfoques transversales a la gestión pública que hablan de los énfasis estratégicos con los cuales se ha decidido emprender el diseño y gestión de las PP de forma democrática y participativa. Así, nos referimos a la participación ciudadana activa, expresada en una política nacional deportiva formulada en un proceso desde abajo hacia arriba, exponiendo las principales problemáticas que la propia población visualiza en sus territorios; la coordinación intersectorial público-privada que, a través de un sistema deportivo nacional formalizado mediante Decreto Presidencial (Chile, 2017), reconfigura el mapa de roles y responsabilidades y busca comprometer el trabajo de organizaciones del ámbito privado y del tercer sector. A lo anterior, se suma la gestión territorial a todo nivel, mediante el vínculo estratégico con el aparato público regional y local (municipalidades) en la implementación de acciones que, desde los territorios, contribuyen a un diagnóstico que es compartido a nivel nacional, como son los datos de (no)práctica de actividad física y sus efectos derivados.

El principio de la gestión territorial permite identificar el segundo avance en las PP del deporte que reconoce este trabajo, relacionado con la innovación propuesta en una estrategia metodológica que contempla la articulación de políticas, planes y programas de fomento deportivo a través de dimensiones, áreas de trabajo y líneas de acciones comunes, que se matizan a nivel territorial con las contribuciones que realizan los municipios y las instituciones regionales en su cumplimiento.

No obstante, dado el nivel de madurez y desarrollo del sistema deportivo nacional actual, la duda cabe en las capacidades y posibilidades institucionales para poner en marcha la implementación de acciones de PP, que no solo requieran de la coordinación del MINDEP, sino que también asuman un rol protagónico otros actores estratégicos (gobiernos regionales y locales), evitando así la lógica de la centralización.

Por otra parte, el horizonte decenal de este instrumento plantea un desafío no menor al sistema político formal, que significa el respeto a la sostenibilidad y trazabilidad de acciones que, probablemente, deban traspasar al menos dos períodos de gobierno para ser evaluadas de forma concreta, 
como el cambio de hábitos y estilos de vida, el aumento de la práctica de actividad física sistemática, el mejoramiento de los resultados en el alto rendimiento, el desarrollo de las organizaciones deportivas, entre otros.

A los anteriores desafíos para el desarrollo de la PP, se suma como factor, en octubre de 2019, el inicio de una aguda crisis política y social en el país, siendo la más crítica y relevante que ha visto Chile en los últimos treinta años y que se origina producto de las consecuencias derivadas de la ideología neoliberal que ha dirigido y orientado la forma de gestionar el Estado. Es desde aquí, donde surgen de forma natural, preguntas y desafíos en torno a analizar de qué forma esta coyuntura impactará la instalación de la reciente PP del sector.

\section{Referencias}

Badilla, C. \& Kemnis, H. (2016). Análisis de la gestión del Ministerio del Deporte. Tesis para optar al grado de Licenciado en Administración Pública. Santiago: Universidad Academia de Humanismo Cristiano. Recuperado de http://bibliotecadigital.academia.cl/handle/ $123456789 / 3934$

Barría, X. (2017). Análisis de la implementación de la Política Nacional de Actividad Física y Deportes a través de la gestión que realizó el IND en los años 2015 y 2016. Memoria para optar al grado de Magíster en Gobierno y Sociedad. Santiago: Universidad Alberto Hurtado. Recuperado de http://repositorio.uahurtado.cl/bitstream/ handle/11242/23790/MGSBarriaV.pdf?sequence=1\&isAllowed=y

Beck, U. (2004). ¿Qué es la globalización? Falacias del globalismo, respuestas a la globalización. Buenos Aires: Paidós.

Bravo, G \& Silva, J. (2013). Sport Policy in Chile, International Journal of Sport Policy and Politics, 6(1), 129-142. Doi: https://doi.org/ 10.1080/19406940.2013.806341

Canales-Lacruz, I. \& Rey-Cao, A. (2018). The Socialization Function of Sport in the Election Manifestos of Spanish Political Parties: General Election 2011. Retos, 33, 233-237. Recuperado de https:/ /recyt.fecyt.es/index.php/retos/article/view/57711/36649

Canan, F., Silva, APJ da., Oliveira, AAB de \& Starepravo, F. (2014). A configuração da rede seti esportes: discutindo e avaliando a partir da policy analysis. Journal of Physical Education, 25(3), 391-403. Doi: http://dx.doi.org/10.4025/reveducfis.v25i3.22185

Castillo, F., Canan, F. \& Starepravo, F. (2017). Políticas Públicas de esporte no Chile: trânsito à democratização. Caderno de Educação Física e Esporte, 15(1), 73-81. Recuperado de http://erevista.unioeste.br/index.php/cadernoedfisica/article/view/15744/ pdf_1

Castillo-Retamal, F., Almonacid-Fierro, A., Castillo-Retamal, M. \& Oliveira, AAB. (2020). Formación de profesores de Educación Física en Chile: una mirada histórica. Retos, 38, 317-324. Recuperado de https://recyt.fecyt.es/index.php/retos/article/view/73304/ 48060

Chile. (1942). Decreto $N^{\circ}$ 35/6331, del 09 de noviembre de 1942. Santiago: Ministerio del Interior. Recuperado de https://www.bcn.cl/ obtienearchivo?id=recursoslegales/10221.3/24639/3/19430521.pdf

Chile. (1942). Ley 7.200, que crea la Dirección de Informaciones y Cultura. Santiago: Ministerio del Interior. Recuperado de https:// www.bcn.cl/obtienearchivo?id=recursoslegales/10221.3/24639/3/ 19430521.pdf

Chile.(1970).Ley 17.276, del 05 de enero de 1970, que crea la DIGEDER. Santiago: Ministerio de Defensa Nacional. Recuperado de https:// www.leychile.cl/Navegar?idNorma $=28882$

Chile. (1971). Decreto $N^{\circ} 369$, del 06 de agosto de 1971, que aprueba el Reglamento relativo a los Consejos Provinciales y Locales de Deportes. Santiago: Ministerio de Defensa Nacional, Subsecretaría de Guerra. Recuperado de https://www.leychile.cl/Consulta/m/ norma plana?org $=\&$ idNorma $=182132$

Chile. (2001). Decreto $N^{\circ 59}$, que establece el Reglamento de Organizaciones Deportivas. Santiago: Ministerio Secretaría General de Go- bierno. Recuperado de https://www.leychile.cl/ Navegar?idNorma $=196442 \&$ idParte $=0$

Chile. (2001). Ley 19.712, del 30 de enero de 2001. Santiago: Ministerio del Interior. Recuperado de https://www.leychile.cl/ Navegar?idNorma $=181636$

Chile. (2002). Política Nacional de Actividad Física y Deporte 20022014. Santiago: Instituto Nacional de Deportes.

Chile. (2006). Decreto $N^{\circ} 42$, del 21 de enero de 2006, que aprueba el Reglamento del Programa de becas para Deportistas de Alto Rendimiento del Instituto Nacional de Deportes. Santiago: Ministerio Secretaría General de Gobierno. Recuperado de https:// www.leychile.cl/Consulta/listado_n_sel?agr=1042

Chile. (2006). Decreto $N^{\circ} 75$, del 03 de agosto de 2006, que aprueba Reglamento sobre Organizaciones Deportivas Profesionales. Santiago: Ministerio Secretaría General de Gobierno. Recuperado de https://www.leychile.cl/Consulta/listado_n_sel?agr=1042

Chile. (2007). Ley 20.178, del 02 de abril de 2007, que regula la relación laboral de los deportistas profesionales y trabajadores que desempeñan actividades conexas. Santiago: Ministerio del Trabajo y Previsión Social. Recuperado de https://www.leychile.cl/ Navegar?idNorma $=260388$

Chile. (2011). Ley 20.500, sobre asociaciones y participación ciudadana en la gestión pública. Santiago: Ministerio Secretaría General de Gobierno de Chile. Recuperado de https://www.leychile.cl/ Navegar?idNorma $=1023143$

Chile. (2012). Encuesta de actividades de niños, niñas y adolescentes. Santiago: Ministerio de Desarrollo Social. Recuperado de http:// educacion2020.cl/documentos/encuesta-de-actividades-de-ninosninas-y-adolescentes-2/

Chile. (2013). Ley 20.686, del 19 de agosto de 2013, que crea el Ministerio del Deporte. Santiago: Ministerio de Secretaría General de Gobierno. Recuperado de https://www.leychile.cl/ Navegar?idNorma $=1053691$

Chile. (2015). Decreto $N^{\circ} 4$, del 03 de julio de 2015, que deroga Decreto №29 de 2012 y aprueba nuevo Reglamento del Programa de becas para Deportistas de Alto Rendimiento. Santiago: Ministerio Secretaría General de Gobierno. Recuperado de https://www.leychile.cl/ Navegar?idNorma $=1083753$

Chile. (2015). Estudio Nacional de Educación Física. Santiago: Agencia de Calidad de la Educación. Ministerio de Educación. Recuperado de http://archivos.agenciaeducacion.cl/ Informe_Nacional_EducacionFisica2015.pdf

Chile. (2016). Política Nacional de Actividad Física y Deporte 20162025. Ministerio del Deporte. Recuperado de http:// www.mindep.cl/wp-content/uploads/2015/05/POLITICA-ULTIMA-VERSI $\%$ C3\% $\% 33 \mathrm{~N}-021116 . p d f$

Chile. (2017). Decreto $N^{\circ} 31$, del 11 de abril de 2017, que aprueba Política Nacional de Actividad Física y Deporte y Crea Comité Interministerial de la Actividad Física y el Deporte. Santiago: Ministerio del Interior. Recuperado de: https://www.leychile.cl/ Navegar?idNorma $=1101746$

Chile. (2017). Encuesta Nacional de Salud 2016-2017. Santiago: Ministerio de Salud. Recuperado de https://www.minsal.cl/wpcontent/uploads/2017/11/ENS-2016-17_PRIMEROSRESULTADOS.pdf

Chile. (2017). Recomendaciones para la práctica de actividad física según curso de vida. Santiago: Gobierno de Chile. Recuperado de http://www.mindep.cl/wp-content/uploads/2016/06/ Recomendaci\%C3\%B3n-para-la-pr\%C3\%Alctica-de-actividadf\%C3\%ADsica-seg\%C3\%BAn-curso-de-vida.pdf

Chile. (2018). Encuesta Nacional de Actividad Física y Deporte en Población de 18 años y más. Santiago: Ministerio del Deporte. Recuperado de http://www.mindep.cl/encuesta-actividad-fisica-ydeporte-2018/

Cornejo, M., Matus, C. \& Vargas, C. (2011). La Educación Física en Chile: una aproximación histórica. EFDeportes Revista Digital,16(161). Recuperado de https://www.efdeportes.com/ efd161/la-educacion-fisica-en-chile.htm

Corporación Participa. (2008). Manual de Participación Ciudadana. Santiago: Corporación Participa. 
De Quadros Martins, D. (2011). Políticas de esporte e as escolhas governamentais. En Júnior, WM: Ensaios em Sociologia do Esporte. Curitiba: Factash Editora.

Díaz, A. (2017). Participación ciudadana en la gestión y en las políticas públicas. Gestión y Política Pública, 26(2), 341-379. Recuperado de http://www.gestionypoliticapublica.cide.edu/ojscide/index.php/ gypp/article/view/337/85

Escobar, H. \& Rivas, G. (2013). Diagnóstico de las políticas públicas deportivas en Chile. Tendencias globales y desafíos para nuestro país. Memoria para optar al grado de Licenciado en Ciencias Jurídicas y Sociales. Santiago: Universidad de Chile. Recuperado de http://repositorio.uchile.cl/bitstream/handle/2250/113270/ deescobar h.pdf? sequence $=1$ \&isAllowed $=\mathrm{y}$

Flick, U. (2015). El diseño de investigación cualitativa. Madrid: Morata. Recuperado de https://dpp2017blog.files.wordpress.com/2017/08/ disec3b1o-de-la-investigacic3b3n-cualitativa.pdf

Flores, Z. (2020). Mujer y deporte en México. Hacia una igualdad sustancial. Retos, 37, 222-226. Recuperado de https://recyt.fecyt.es/ index.php/retos/article/view/71684/45234

Frey, K. (2000). Políticas Públicas: um debate conceitual e reflexões referentes à prática da análise de Políticas Públicas no Brasil. Planejamento e Políticas Públicas, 21, 211-259. Recuperado de http://www.ipea.gov.br/ppp/index.php/PPP/article/view/89/158

Gil, A. (2002). Como elaborar projetos de pesquisa. São Paulo: Atlas. Recuperado de http://www.urca.br/itec/images/pdfs/ modulo $\% 20 \mathrm{v} \% 20 \% 20$ como_elaborar_projeto_de_pesquisa_antonio carlos gil.pdf

Jofré, I. (2014). Desarrollo de la Actividad Física y Deportiva, Fomento Público y Responsabilidad Social Empresarial. Memoria para optar al grado de Licenciado en Ciencias Jurídicas y Sociales. Santiago: Universidad de Chile. Recuperado de http:// repositorio.uchile.cl/bitstream/handle/2250/115608/dejofre i.pdf?sequence $=1 \&$ isAllowed $=y$

Marcen, C., Murillo, R., Arbones, I. \& Pelegrín, J. (2019). Countercultural sports and local policies: an approach from the theory of public policy networks. Retos, 35, 377-382. Recuperado de https:// recyt.fecyt.es/index.php/retos/article/view/65893

Matus, C., Vilanova, A., Puig, N. \& Vidal, J. (2018). Las etapas del asociacionismo deportivo en Chile y su relación con el contexto histórico (finales siglo XIX-2012). RICYDE. Revista internacional de ciencias del deporte, 53(14), 280-296. Doi: https://doi.org/ 10.5232/ricyde2018.05308

Moreno, A. \& Poblete, C. (2015). La educación física chilena y su profesorado: proponiendo algunos retos para la investigación en el área. Retos, 28, 291-296. Recuperado de https://recyt.fecyt.es/ index.php/retos/article/view/35651/19677

Moreno, R. (2015). Políticas públicas y deporte. Un análisis de la municipalización deportiva en Lerma, Estado de México. Revista Electrónica del Centro de Estudios en Administración Pública, 21, 1-20. Doi: http://dx.doi.org/10.22201/ fcpys.20071949e.2015.21.57646

Mujica, P. (2005). La participación ciudadana en relación con la gestión pública. Santiago: Corporación Participa.

Muñoz, C. (2001). Historia de la Dirección General de Deportes y Recreación. Las Políticas Estatales de Fomento al Deporte: DIGEDER 1948-2001. Santiago: Instituto Nacional de Deportes de Chile.

Organización de las Naciones Unidas para la Educación, la Ciencia y la Cultura - UNESCO. (2013). Declaración de Berlín. Berlín: MINEPS V. Recuperado de https://unesdoc.unesco.org/ark:/48223/ pf0000221114_spa

Organización de las Naciones Unidas para la Educación, la Ciencia y la Cultura - UNESCO. (2015). Carta Internacional de la Educación Física, la Actividad Física y el Deporte. Recuperado de http:// $\mathrm{p}$ o r t a 1 . u n e s c o. o r g / e s/e v.p h p URL ID=13150\&URL DO=DO TOPIC\&URL_SECTION=201.html

PNUD. (2004). La democracia en América Latina. Hacia una democracia de ciudadanos y ciudadanas. Buenos Aires: Alfaguara. Recuperado de http://www.bantaba.ehu.es/obs/ocont/pd/obsdoc/ demamerlat/
Putnam, R. (1993). Making Democracy Work. Civic Traditions in Modern Italy. Princeton: Princeton University Press.

Quivy, R. \& Van Campenhoudt, L. (2006). Manual de investigación en Ciencias Sociales. México: Limusa.

Rodríguez, J. (2017). Políticas Públicas. Revista Venezolana de Enfermería, 4(2), 17-26. Recuperado de http://saber.ucv.ve/ojs/index.php/ rev_venf/article/view/15917/144814482540

Roth, A. (2014). Políticas públicas: formulación implementación y evaluación. $10^{\circ}$ edición. Bogotá: Aurora

Sabatini, F., Arenas, F. \& Núñez, A. (2011). Identidades territoriales en Chile: entre la globalización y el regionalismo. Scripta Nova, Revista Electrónica de Geografía y Ciencias Sociales, 15(379). Recuperado de http://www.ub.edu/geocrit/sn/sn-379.htm

Salazar, G (1997). Descentralización y sinergia histórica Local: Fracasos y Desafíos. Cyber Humanitatis, 4, 1-14. Recuperado de https:/ /cyberhumanitatis.uchile.cl/index.php/RCH/article/view/27858/ 29533

Sandoval, P. \& García, I. (2014). Cultura deportiva en Chile: desarrollo histórico, institucionalidad actual e implicancias para la política pública. Polis, 13(39), 441-462. Doi: http://dx.doi.org/10.4067/ S0718-65682014000300020

Segura, F. \& Buarque de Hollanda, B. (2015). El estudio del deporte y las políticas públicas. Gestión y Política Pública, vol. temático 2015, 3-25. Recuperado de http://www.gestionypoliticapublica.cide.edu/ ojscide/index.php/gypp/article/view/117/41

Soto-Lagos, R. (2016). Sedentarismo, deporte y la presión biopolítica de vivir saludable: análisis del discurso del sistema Elige Vivir Sano en Chile. Movimento, 22(2), 391-402. Recuperado de https:// seer.ufrgs.br/Movimento/article/viewFile/57338/37372

Soto-Lagos, R. (2018). Deporte, prácticas corporales, vida saludable y buen vivir: un análisis crítico para una nueva praxis. ALESDE Revista Latinoamericana de Estudios Socioculturales del Deporte, 9(1), 29-44. Recuperado de https://revistas.ufpr.br/alesde/article/ view/61305/36045

Taibo, C. \& Palacio, P. (2017). Análisis de la implementación de la política pública deportiva: el caso del programa de deporte y participación social en la Región del Biobío, año 2016-2017. Seminario para optar al grado de Administrador Público. Concepción: Universidad de Concepción. Recuperado de http:// administracionpublica.udec.cl/wp-content/uploads/2018/04/TesisCarlos-Taibo-y-Patricio-Palacio.pdf

Tejero-González, C. (2016). Sobre la importancia del deporte como acción política: Razones y medidas de gobernanza. Cultura, Ciencia y Deporte, 11(31), 5-6. Recuperado de http://www.redalyc.org/ pdf/1630/163044427001.pdf

Valdebenito, D. (2013). Análisis crítico del modelo deportivo nacional a la luz del derecho comparado. Memoria para optar al grado de Licenciado en Ciencias Jurídicas y Sociales. Santiago: Universidad de Chile. Recuperado de http://repositorio.uchile.cl/bitstream/ $\mathrm{h}$ a n d $1 \mathrm{e} / 2 \begin{array}{llllllllllll}2 & 5 & 0 & 1 & 1 & 3 & 2 & 3 & 1 & / \mathrm{d} & \mathrm{e}\end{array}$ valdebenito_d.pdf? sequence $=1 \&$ isAllowed $=\mathrm{y}$

Valdez, S. (2015). Fomento de la actividad física en México. Una política pública inacabada. Gestión y Política Pública, vol. temático 2015, 27-54. Recuperado de http:// www.gestionypoliticapublica.cide.edu/ojscide/index.php/gypp/ article/view/118/42

Vargas-Contreras, C. (2015). ¿Cuándo, cómo y dónde?: Análisis de la pertinencia, relevancia y aplicabilidad de la participación ciudadana en la gestión pública del sector deporte. Estudio de caso del Programa Deporte de Participación Social del Ministerio del Deporte. Memoria para optar al grado de Magíster en Gobierno y Sociedad. Santiago: Universidad Alberto Hurtado. Recuperado de http://repositorio.uahurtado.cl/bitstream/handle/11242/8002/ MGSVargasC.pdf? sequence $=2$ \&isAllowed $=y$

Vargas-Contreras, C., Matus-Castillo, C. \& Duclos-Bastías, D. (2020). Las políticas públicas para la promoción de la actividad física y el deporte en Chile y su relación con los hábitos de vida saludable y la calidad de vida. En Poblete-Valderrama, F. (Ed.), Actividad Física y Salud: «enfoques, reflexiones y evidencias». Santiago, Chile: RIL Editores. (en edición) 